\title{
PASSIVELY MODE-LOCKED Nd-GLASS LASER WITH PARTIALLY SUPPRESSED NATURAL MODE SELECTION
}

\author{
F. GRAF, C. LÖW and A. PENZKOFER \\ Naturwissenschaftliche Fakultät II, Physik, Universität Regensburg, \\ 8400 Regensburg, Fed. Rep. Germany
}

Received 20 June 1983

\begin{abstract}
The performance of a passively mode-locked Nd-glass laser with an intracavity solid etalon is studied. For a $0.1 \mathrm{~mm}$ thick uncoated fused silica plate maximal spectral broadening (factor 4 to 5) and temporal pulse shortening (factor 1.5 to 2 ) is achieved at a tilting angle of $\approx 43^{\circ}$. Theoretical analysis of temporal pulse development agrees with experimental findings.
\end{abstract}

\section{Introduction}

The spectral narrowing of emission in the linear region of passively mode-locked lasers is thought to increase the final pulse duration obtained in the nonlinear mode-locking region. Properly adjusted FabryPerot interferometers were applied for partial suppression of natural mode-selection in mode-locked ruby $[1,2] \mathrm{Nd}$-glass $[3,4]$, and Nd-YAG lasers [5] . Pulse shortening factors between 1.5 and 3 have been achieved.

In this letter the natural mode selection of a modelocked $\mathrm{Nd}$-glass laser is partially suppressed by inserting a $0.1 \mathrm{~mm}$ thick uncoated fused silica etalon into the laser oscillator. A large tilting angle around $43^{\circ}$ is used to adjust the etalon transmission to the gain curve of the active medium. Fine angle tuning allows to position the transmission minimum of the etalon to the gain maximum of the active medium in order to flatten the net gain distribution. The spectra at the end of the linear phase are broadened by a factor of 4 to 5 . The final pulse durations are shortened by factors between 1.5 and 2.

The pulse shortening is limited by reduced suppression of spikes within the opening time of the modelocking dye and by pulse broadening due to two-photon absorption in the active medium at elevated intensities.

The experimental findings are compared with theo- retical calculations of the temporal pulse development in the mode-locking region. Good agreement between theory and experiment is found.

\section{Spectral narrowing in linear laser region}

The spectral gain profile of the active medium narrows the spectral emission in repetitive transits within the linear region of the mode-locked laser. The net gain per round-trip is given by

$G(\nu)=G_{\mathrm{A}}(\nu) T_{\mathrm{F}}^{2}(\nu) R_{1} R_{2} T_{1} T_{0}^{2}$.

$G_{\mathrm{A}}(\nu)$ is the gain of the active medium per round-trip. $T_{\mathrm{F}}$ represents the single-pass transmission through the etalon. $R_{1}$ and $R_{2}$ are the reflectivities of the laser mirrors. $T_{1}$ accounts for linear losses due to diffraction, scattering and absorption. $T_{0}$ is the small-signal single-pass transmission of the saturable dye.

The laser transition is inhomogeneously broadened and $G_{\mathrm{A}}(\nu)$ is approximately given by

$G_{\mathrm{A}}(\nu)=\exp \left[2 l_{\mathrm{A}} \sigma_{\mathrm{A}} N_{\mathrm{U}}(\nu)\right]$.

$l_{\mathrm{A}}$ is the length of the pumped region of the active medium. $\sigma_{\mathrm{A}}$ is the effective stimulated emission cross section (for details see ref. [6]). $N_{\mathrm{U}}(\nu)$ is the number density of $\mathrm{Nd}^{3+}$ ions excited to the upper laser level. The population of the lower laser level is neglected since it decays rapidly to the ground state [7]. The 
spectral distribution of $N_{\mathrm{U}}(\nu)$ is approximately gaussian, i.e. $N_{\mathrm{U}}(\nu)=N_{\mathrm{U} 0} \exp \left[-\left(\nu-\nu_{0}\right)^{2} / \nu_{\mathrm{G}}^{2}\right]$, where $\nu_{0}$ is the central laser frequency and $\nu_{\mathrm{G}}$ is the $1 / \mathrm{e}$-width of the inhomogeneous distribution.

The single-pass transmission through the etalon is [8]

$$
\begin{aligned}
& T_{\mathrm{F}}(\nu)=\left(1-R_{\mathrm{F}}\right)^{2}\left\{\left(1-R_{\mathrm{F}}\right)^{2}\right. \\
& \left.\quad+4 R_{\mathrm{F}} \sin ^{2}\left[2 \pi \nu d\left(n_{\mathrm{F}}^{2}-n_{\mathrm{A}}^{2} \sin ^{2} \alpha\right)^{1 / 2} / c\right]\right\}^{-1} .
\end{aligned}
$$

$R_{\mathrm{F}}$ is the reflection coefficient of the Fabry-Perot etalon. Its value decreases with increasing tilting angle $\alpha$ for p-polarized light according to $R_{\mathrm{F}}=\left[\tan \left(\alpha-\alpha^{\prime}\right) /\right.$ $\left.\tan \left(\alpha+\alpha^{\prime}\right)\right]^{2}$ where $\alpha^{\prime}=\arcsin \left[n_{\mathrm{A}}(\sin \alpha) / n_{\mathrm{F}}\right.$ ] [9] . $d$ is the thickness of the plate and $n_{\mathrm{F}}$ its absolute refractive index. $n_{\mathrm{A}}$ is the absolute refractive index of air. The transmission varies with frequency $\nu$ and angle $\alpha$ between $T_{\mathrm{F}}=1$ and $T_{\mathrm{F}}=T_{\min }=\left(1-R_{\mathrm{F}}\right)^{2} /\left(1+R_{\mathrm{F}}\right)^{2}$. At a fixed angle $\alpha$ the free spectral range (frequency spacing between two adjacent transmission minima) is $\Delta \nu_{\mathrm{F}}=c / 2 d\left(n_{\mathrm{F}}^{2}-n_{\mathrm{A}}^{2} \sin ^{2} \alpha\right)^{1 / 2}$. Eq. (3) is exactly valid only for infinite temporal and spatial pulse extension. It remains a good approximation for pulse duration $\Delta t_{\mathrm{L}}>2 n_{\mathrm{F}} d / c(=1 \mathrm{ps}$ in our case $)$ and beam radius $r>2 d \tan (\alpha) / n_{\mathrm{F}}\left(=0.1 \mathrm{~mm}\right.$ for $d=0.1 \mathrm{~mm}, n_{\mathrm{F}}=1.5$ and $\alpha=43^{\circ}$ ).

The net gain curve $G(\nu)$ is optimally flattened around $\nu_{0}$ when the solid etalon is adjusted to

$$
\partial^{2} T_{\mathrm{F}}^{2}\left(\nu_{0}, \alpha\right) / \partial \nu^{2}=-\partial^{2} G_{\mathrm{A}}\left(\nu_{0}\right) / \partial \nu^{2},
$$

i.e. the curvature of the gain curve of the active medium at $\nu_{0}$ should be compensated by the opposite curvature of the etalon transmission. Condition (4) is approximately satisfied by adjusting the tilting angle $\alpha$ to obtain proper modulation depth $T_{\min }$ (coarse tuning) and to position the transmission minimum to frequency $\nu_{0}$ (fine tuning).

In fig. 1a calculated gain profiles are presented. The solid curve shows the net gain per round-trip without etalon $\left(G\left(\nu_{0}\right)=1.08\right)$. The used parameters are listed in the figure captions. The dashed curve depicts the frequency dependence of $T_{\mathrm{F}}^{2}(\nu)$ at $\alpha=49.5^{\circ}$. The dotted curve gives the resulting net gain of the resonator with intracavity etalon. The flattening of the gain curve around $\nu_{0}$ is clearly seen.

In fig. $1 \mathrm{~b}$ spectral distributions are displayed. Curve 1 exhibits the fluorescence spectrum of the active medium approximated by a gaussian distribution (used in
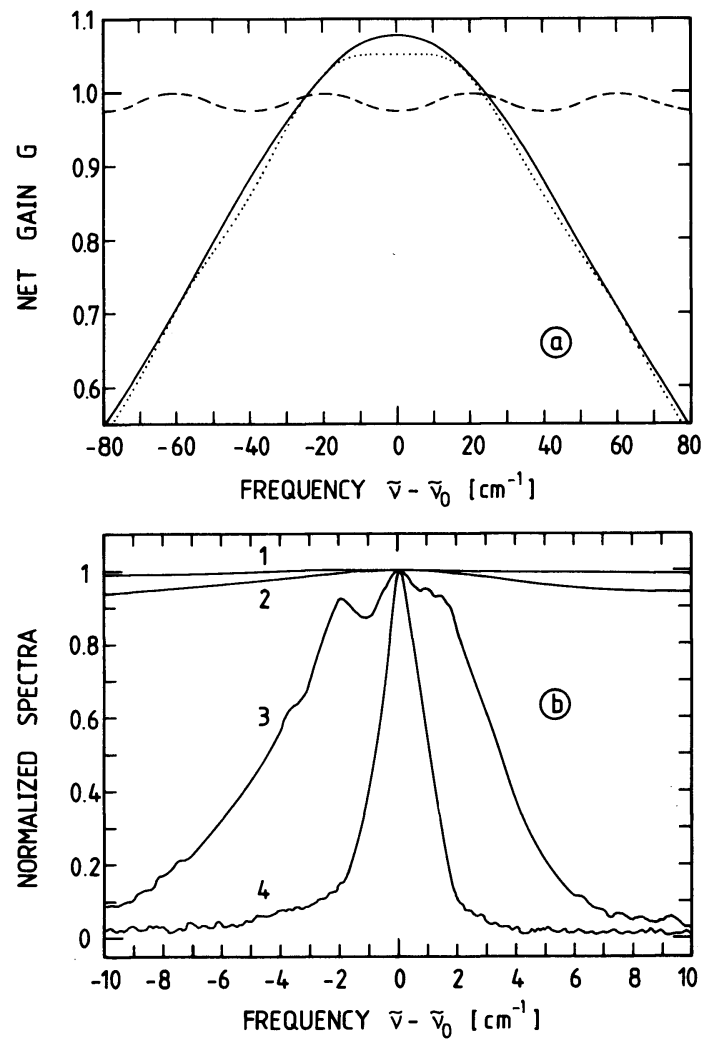

Fig. 1. Spectral changes by solid etalon. (a) Dashed curve: Double pass transmission of solid etalon $T_{\mathrm{F}}^{2}$ [eq. (3)] . Thickness $d=0.1 \mathrm{~mm}$, tilting angle $\alpha=49.5^{\circ}$, refractive indices $n_{\mathrm{F}}$ $=1.4501, n_{\mathrm{A}}=1.0002678, \tilde{\nu}_{0}=\nu_{0} / c=9496.7 \mathrm{~cm}^{-1}$. Solid curve: Net gain $G(\nu)$ per round-trip without etalon [eq. (1)]. Gain adjusted to $G\left(\nu_{0}\right)=1.08$. Used parameters are $R_{1}$ $=0.997 . R_{2}=0.3, T_{1}=0.9, T_{0}=0.85, l_{\mathrm{A}}=10 \mathrm{~cm}, \sigma_{\mathrm{A}}=4.1$ $\times 10^{-20} \mathrm{~cm}^{2}, \tilde{\nu}_{\mathrm{G}}=\nu_{\mathrm{G}} / c=113 \mathrm{~cm}^{-1}$. Dotted curve: Net gain per round-trip with etalon (product of dashed and solid curve). (b) Curve 1: Gaussian fluorescence profile with 1/e-width $\widetilde{\nu}_{G}$ $=113 \mathrm{~cm}^{-1}$. Curve 2: Experimental spectral distribution of fluorescence emission. Curve 3: Spectrum of free running laser with properly adjusted etalon. $\alpha=43.4^{\circ}$. Curve. 4 : Spectrum of free running laser without etalon.

fig. 1a). Curve 2 shows the measured fluorescence emission of the $\mathrm{Nd}$-glass rod (proportional to $N_{\mathrm{U}}(\nu)$ ). It reveals a rippling which enhances spectral narrowing. Curve 3 presents the spectrum of the free running laser (no mode-locking dye in oscillator) without solid etalon. The spectral width is $\Delta \widetilde{\nu}=\Delta \nu / c=1.9 \mathrm{~cm}^{-1}$ (fwhm). The spectrum of the free-running laser with properly adjusted etalon $\left(\alpha=44.9^{\circ}\right)$ is shown by curve 4. Its spectral width is $\Delta \widetilde{\nu}=8 \mathrm{~cm}^{-1}$. 


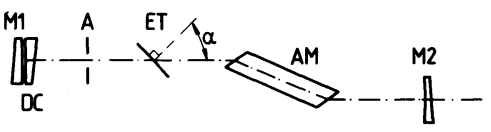

Fig. 2. Passively mode-locked Nd-glass laser oscillator. M1, plane mirror with $R_{1}=0.997$ reflectivity. $\mathrm{M} 2$, mirror of $3 \mathrm{~m}$ curvature and $R_{2}=0.3$. DC, contacted dye cell. $\mathrm{A}$, aperture. $\mathrm{ET}$, solid etalon. AM, active medium (Nd-phosphate glass rod).

\section{Experimental}

The experimental arrangement of the laser oscillator is depicted in fig. 2. The hemiconfocal resonator is formed by two mirrors M1 $\left(R_{1}=0.997\right.$, plane $)$ and M2 $\left(R_{2}=0.3\right.$, curvature $\left.3 \mathrm{~m}\right)$. A Brewster-Brewster wedged Nd-phosphate glass rod (Schott LG703, 3 weight $\% \mathrm{Nd}_{2} \mathrm{O}_{3}$ ) of $l_{\mathrm{R}}=13 \mathrm{~cm}$ length is used (pumped length $l_{\mathrm{A}}=10 \mathrm{~cm}$ ). The mode-locking dye Eastman 9860 is circulated through a contacted cell of $0.1 \mathrm{~mm}$ thickness (single-pass dye transmission $T_{0}$ $=0.85$ ). The aperture A confines the transversal mode structure to $\mathrm{TEM}_{00}$. The solid etalon is a $0.1 \mathrm{~mm}$ thick uncoated fused silica plate (flatness $\lambda / 10$ ). It is mounted on a rotation stage for angle tuning. At the used tilting angle of $\alpha=43.4^{\circ}$ the free spectral range of the etalon is $\Delta \widetilde{\nu}_{\mathrm{F}}=39 \mathrm{~cm}^{-1}$. An angle tuning of $\Delta \alpha=0.77^{\circ}$ causes a spectral shift of $\Delta \widetilde{\nu}_{\mathrm{F}}$.

Single picosecond pulses along the pulse train are selected with a krytron triggered Pockels cell shutter. The spectra are measured with a $60 \mathrm{~cm}$ grating spectrograph (1200 lines/mm, spatial dispersion $0.9 \mathrm{~nm} /$ $\mathrm{mm}$ ) and recorded with a silicon vidicon. The durations of the pulses are measured after pulse amplification in a Nd-glass amplifier by two-photon fluorescence detection (dye $2.5 \times 10^{-3}$ molar rhodamine $6 \mathrm{G}$ in ethanol). The fluorescence traces are recorded with an intensified silicon vidicon.

\section{Results}

Spectral and temporal pulse shapes are illustrated in fig. 3. The picosecond pulses are selected in the rising part of the pulse train at a height of $\approx 0.2$ the height at the train maximum. Early pulse selection avoids the problems of self-phase modulation [10] and spectral gain depletion [11]. The spectrum (a) and the

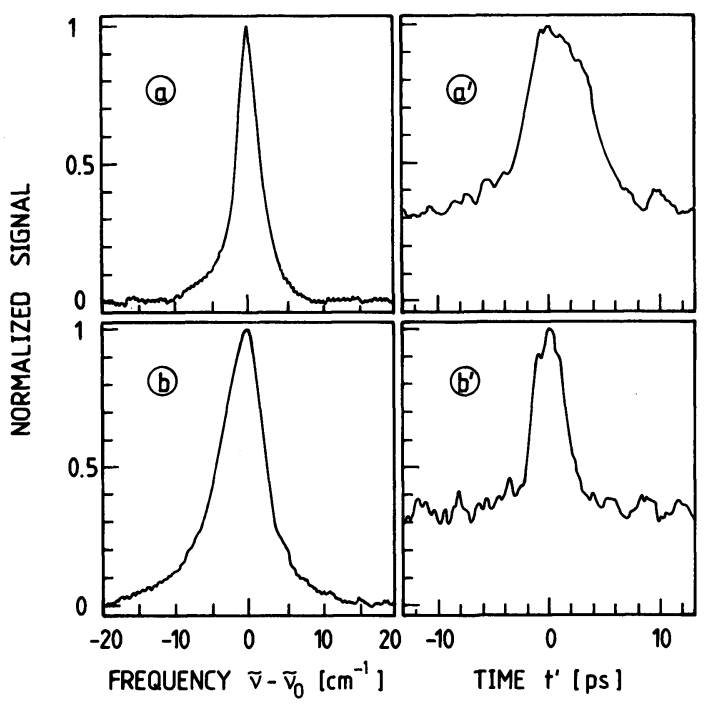

Fig. 3. Single-pulse spectra and two-photon fluorescence traces selected in rising part of pulse train. Time scale is adjusted so that half-width of traces corresponds to duration of gaussian shaped pulses. (a), (a') without etalon: $\Delta \tilde{\nu}_{\mathrm{L}}=3.4$ $\mathrm{cm}^{-1}$ (fwhm), $\Delta t_{\mathrm{L}}=6.7 \mathrm{ps}$ (fwhm). (b), (b') with etalon: $\Delta \tilde{\nu}_{\mathrm{L}}=7 \mathrm{~cm}^{-1}, \Delta t_{\mathrm{L}}=3.4 \mathrm{ps}$.

two-photon fluorescence trace $\left(\mathrm{a}^{\prime}\right)$ belong to a laser pulse generated without etalon. Its spectral width is $\Delta \tilde{\nu}_{\mathrm{L}}=3.4 \mathrm{~cm}^{-1}(\mathrm{fwhm})$ and its duration is $\Delta t_{\mathrm{L}}=6.7$. ps (fwhm). Figs. 3b and 3b' depict a spectrum and a two-photon fluorescence trace of a pulse generated with properly adjusted etalon in the resonator. The spectrum has a width of $\Delta \tilde{\nu}_{\mathrm{L}}=7 \mathrm{~cm}^{-1}$, and the pulse duration is $\Delta t_{\mathrm{L}}=3.4 \mathrm{ps}$. Occasionally the two-photon fluorescence trace reveals the presence of two closely spaced picosecond pulses. The secondary pulse lies within the opening time of the mode-locking dye (recovery time $\tau_{R}=7 \mathrm{ps}[11]$ ). This occasional occurrence of double pulses is expected since statistical spikes originally present are not sufficiently discriminated within the opening time of the absorber in the nonlinear mode-locking region. Insertion of a saturable absorber (Eastman 9860) of small-signal transmission $T=5 \times 10^{-3}$ behind the oscillator suppressed the secondary pulses and shortened the pulse envelope by approximately a factor of 1.5 . 


\section{Temporal pulse development in nonlinear laser region.}

In the experiments with the etalon we observed a shortening of the pulse duration by a factor of 1.5 to 2 while the spectral width at the end of the linear phase was broadened by a factor of 4 to 5 . The following theoretical analysis explains the reduced pulse shortening by an interplay of pulse shortening in the saturable absorber and pulse broadening in the active medium. The broadening in the laser medium is due to its finite spectral gain width and due to two-photon absorption of $\mathrm{Nd}^{3+}$ ions at elevated intensities [13]

We study the temporal pulse development in the nonlinear phase of the mode-locking laser for the most intense spike. Its intensity distribution at the end of the linear phase is assumed to be

$I_{1}\left(r, t^{\prime}\right)=I_{10} \exp \left(-t^{\prime 2} / t_{0}^{2}\right) \exp \left(-r^{2} / r_{0}^{2}\right)$,

with $t_{0}=\Delta t_{\mathrm{L} 0} /\left[2(\ln 2)^{1 / 2}\right]$ and $\Delta t_{\mathrm{L} 0} \approx 0.5 / \Delta \nu_{\mathrm{L} 0}$ $\left(I_{10}=10^{6} \mathrm{~W} / \mathrm{cm}^{2}, \Delta \widetilde{\nu}_{\mathrm{L} 0}=8 \mathrm{~cm}^{-1}\right.$ with etalon, $\Delta \widetilde{\nu}_{\mathrm{L} 0}$ $=1.9 \mathrm{~cm}^{-1}$ without etalon. At the $j$ th round trip in the nonlinear phase the intensity distribution $I_{j}\left(t^{\prime}\right)$ changes to

$I_{j+1}\left(t^{\prime}\right)=T_{\mathrm{d}, j}\left(t^{\prime}\right) T_{\mathrm{TPA}, j}\left(t^{\prime}\right) G_{j} \int_{-\infty}^{t^{\prime}} I_{j}(t) g\left(t-t^{\prime}\right) \mathrm{d} t$.

$T_{\mathrm{d}, j}\left(t^{\prime}\right)$ is the transmission of the saturable absorber. $T_{\mathrm{TPA}, j}\left(t^{\prime}\right)$ accounts for two-photon absorption in the active medium. $G_{j}$ is the net gain per round-trip without saturable absorber and two-photon absorption losses. $g\left(t-t^{\prime}\right)$ is the response function of the active medium.

The saturable absorber may be approximated by a three-level system consisting of a ground state 1 , a fast intermediate state 2 and a relaxed level 3 . The total pulse transmission through the dye in a cell contacted to the $100 \%$ mirror is

$$
\begin{aligned}
& T_{\mathrm{d}, j}\left(t^{\prime}\right)=\exp \left\{-2 \sigma\left[N-\kappa N_{3, j}\left(t^{\prime}\right)\right]\right\} \\
& \quad=T_{0}^{2} \exp \left[2 \kappa \sigma N_{3, j}\left(t^{\prime}\right)\right] .
\end{aligned}
$$

$T_{0}=\exp (-\sigma N)$ is the small-signal single-path transmission. $N$ is the total number density of dye molecules integrated over the cell length. $\sigma$ denotes the isotropic absorption cross section. The correction factor $\kappa$ takes care of the absorption anisotropy of electric dipole in- teraction [14] in an approximative manner $(\kappa=2$ in our calculation).

The population $N_{3, j}\left(t^{\prime}\right)$ is given by

$$
\frac{\mathrm{d} N_{3, j}}{\mathrm{~d} t^{\prime}}=\frac{f I_{j}\left(t^{\prime}\right)}{h \nu_{\mathrm{L}}}\left[1-T_{\mathrm{d}, j}\left(t^{\prime}\right)\right]-\frac{N_{3, j}}{\tau_{\mathrm{R}}} \text {. }
$$

The first term represents the rate of light absorption. The second term is responsible for relaxation of excited molecules. $f$ denotes the increase of intensity in the contacted dye cell compared to the active medium due to reduced beam cross section outside the double Brewster rod (factor $f_{1} \approx 1.5$ ), due to reduced spot size at the plane mirror (factor $f_{2} \approx 1.4$ ) and due to pulse reflection at the mirror (factor $f_{3} \approx 3$ [15]). The resulting factor is $f=f_{1} f_{2} f_{3} \approx 6.5$. The solution of eq. (7) gives

$$
N_{3, j}\left(t^{\prime}\right)=\int_{-\infty}^{t^{\prime}} \frac{f I_{j}(t)}{h \nu_{\mathrm{L}}}\left[1-T_{\mathrm{d}, j}(t)\right] \exp \left[-\left(t^{\prime}-t\right) / \tau_{\mathrm{R}}\right] \mathrm{d} t .
$$

Insertion of eq. (8) into eq. (6) gives an implicit equation for the temporal transmission $T_{\mathrm{d}, j}\left(t^{\prime}\right)$ which is solved numerically.

The two-photon absorption introduces an intensitydependent loss factor that broadens the pulse duration. The transmission per round-trip is

$$
T_{\mathrm{TPA}, j}\left(t^{\prime}\right)=\frac{1}{1+\alpha^{(2)} 2 l_{\mathrm{R}} I_{j}\left(t^{\prime}\right)} .
$$

In the experiments we determined a two-photon absorption cross section of $\alpha^{(2)}=(4 \pm 1) \times 10^{-12} \mathrm{~cm} / \mathrm{W}$ for the applied $\mathrm{Nd}$-phosphate laser rod.

The response function $g(t)$ tends to broaden the pulse duration. It is given by the Fourier transform of the gain profile of the active medium (fig. 1a). For a gaussian profile of spectral width $\Delta \nu_{\mathrm{G}}$ the response function is given by $g(t)=\exp \left[-4(\ln 2) t^{2} / \Delta t_{\mathrm{G}}^{2}\right]$ with $\Delta t_{\mathrm{G}}=0.44 / \Delta \nu_{\mathrm{G}}$. The gain curve of fig. 1a has a spectral half width of $\Delta \widetilde{\nu}_{\mathrm{G}}=163 \mathrm{~cm}^{-1}$ and the corresponding response time is $\Delta t_{\mathrm{G}}=0.09 \mathrm{ps}$. The pulse broadening contribution of the active medium per round-trip is approximated in the calculations by the factor $\xi=\left(\Delta t_{\mathrm{L}, j}^{2}+\Delta t_{\mathrm{G}}^{2}\right)^{1 / 2} / \Delta t_{\mathrm{L}, j}$ for $\Delta t_{\mathrm{L}, j}<\Delta t_{\mathrm{L} 0}$ and $\xi=1$ for $\Delta t_{\mathrm{L}, j+1} \geqslant \Delta t_{\mathrm{L} 0}\left(\Delta t_{\mathrm{L} 0}=9\right.$ ps without etalon, $\Delta t_{\mathrm{L} 0}=2 \mathrm{ps}$ with etalon).

In fig. 4 some results of the calculations are presented. The calculations start at the end of the linear phase. The net gain $G_{j}$ is set to $G_{j}=1.03 / T_{0}^{2}$ until the 


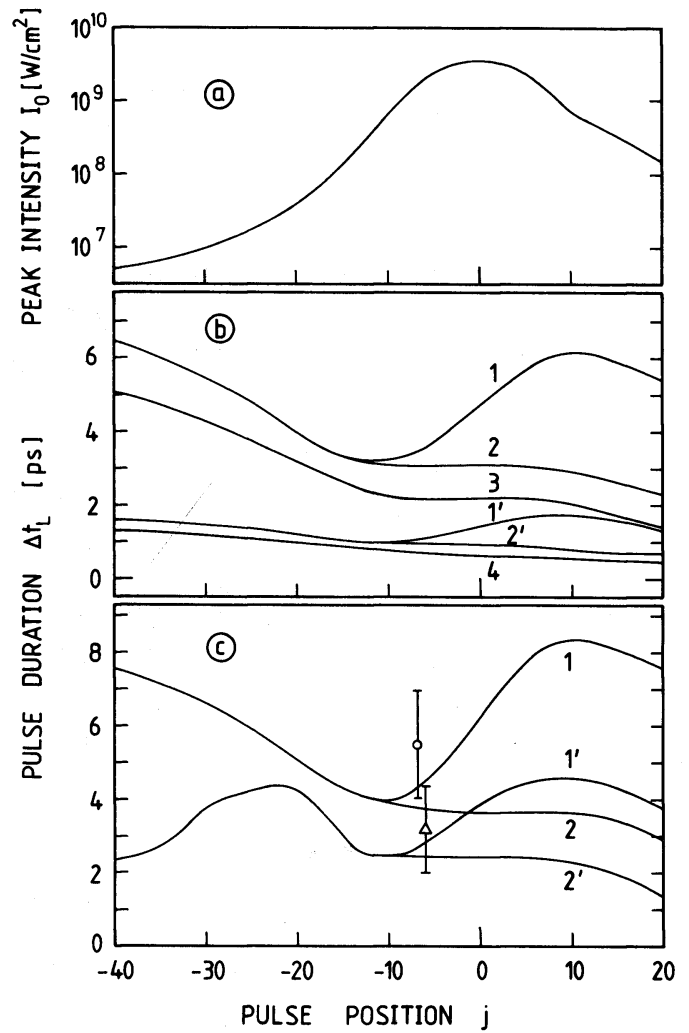

Fig. 4. Theoretical temporal pulse development along pulse train. (a) Peak pulse intensity distribution for situation of fig. 4c, curve 1. (b) Single-pulse development without background. Curve 1, without etalon, with two-photon absorption (TPA). Curve 2, without etalon, without TPA. Curve 1', with etalon and TPA. Curve 2 ', with etalon, without TPA. Curve 3, like curve 2 but $\tau_{\mathrm{R}}=2.7 \mathrm{ps}$ and $\sigma=3 \times 10^{-16} \mathrm{~cm}^{2}$ (data of dye Eastman No. 5 [12]). Curve 4, like curve 2', but without spectral narrowing $\xi=1\left(\Delta t_{\mathrm{G}}=0\right)$. (c) Single-pulse development with background. The peak pulse to background ratio is $\beta=7$ without etalon and $\beta=8.5$ with etalon. Otherwise the same parameter as in (b) are used. Experimental points: circle (O), without etalon; triangle $(\Delta)$, with etalon. Unless otherwise stated the applied parameters of the curves are: Initial pulse duration $\Delta t_{\mathrm{LO}}=9 \mathrm{ps}$ (without etalon) and $\Delta t_{\mathrm{LO}}=2 \mathrm{ps}$ (with etalon), two-photon absorption coefficient $\alpha^{(2)}=4 \times 10^{-12}$ $\mathrm{cm} / \mathrm{W}$, rod length $l_{\mathrm{R}}=13 \mathrm{~cm}$ absorber recovery time $\tau_{\mathrm{R}}=7$ ps, absorption cross section of absorber $\sigma=3.7 \times 10^{-16} \mathrm{~cm}^{2}$, response time of active medium $\Delta t_{\mathrm{G}}=0.09 \mathrm{ps}$.

peak pulse intensity reaches a fifth of the value $I_{0, \max }$ at the train maximum. Then the shape of the pulse train is approximated by a sine shape covering a total of 20 pulses,

$I_{0, m+i}=I_{0, m}+\left(I_{0, \max }-I_{0, m}\right) \sin (\pi i / 20)$.
On the trailing part of the pulse train the net gain is set to $G_{j}=1.03^{-1} / T_{0}^{2}$. In the calculations the peak intensity of the pulse train is reduced a factor of two compared to the experimental value of $I_{0, \max }=4$ $X 10^{9} \mathrm{~W} / \mathrm{cm}$ in order to account for the neglection of integration over the beam cross section.

Fig. 4a shows the shape of the pulse train in case of $\Delta \widetilde{\nu}_{\mathrm{L} 0}=1.9 \mathrm{~cm}^{-1}$ (without etalon) and $\alpha^{(2)}=4$ $\times 10^{-12} \mathrm{~cm} / \mathrm{W}$. In fig. $4 \mathrm{~b}$ the pulse duration of a single pulse without background along the pulse train position is plotted. Curve 2 is responsible for a resonator without etalon and without two-photon absorption. The pulse duration shortens in the early part of the pulse train. Towards the pulse train maximum the shortening levels off since the mode-locking dye becomes completely bleached and looses its shortening action. Curve 1 includes the two-photon absorption. The pulse durations are broadened around the pulse train maximum where two-photon absorption is strong. Curve $1^{\prime}$ is calculated for a resonator with etalon and with two-photon absorption. The pulse duration starts at a lower value. The two-photon absorption broadens the pulse durations around the train maximum. Curve $2^{\prime}$ is calculated without two-photon absorption. The pulse shortens from an initial duration of 2 ps to a duration of $\approx 1 \mathrm{ps}$. The temporal broadening of the active medium acts against further narrowing by the saturable absorber. The influence of the recovery time of the saturable absorber on the final pulse duration is indicated by curve 3 which belongs to $\tau_{\mathrm{R}}=2.7 \mathrm{ps}, \Delta t_{\mathrm{L} 0}$ $=9 \mathrm{ps}$ and $\alpha^{(2)}=0$. A shortening of $\tau_{\mathrm{R}}$ by a factor of 2.6 gives a pulse shortening by a factor of 1.4. The effect of the finite spectral width of the active medium on the pulse duration is illustrated by curve 4 where the broadening effect is neglected. The curve is calculated for $\tau_{\mathrm{R}}=7 \mathrm{ps}, \Delta t_{\mathrm{L} 0}=2 \mathrm{ps}, \alpha^{(2)}=0$ and $\xi=1$.

In fig. $4 \mathrm{c}$ the propagation of a single pulse with constant background intensity is considered. The background level is set equal to the ratio of the mean spike intensity to the intensity of the most intense spike. This ratio is given by $\beta=I_{\mathrm{p}} /\langle I\rangle \approx \ln (2 m)$ with $m=T / 2 \Delta t_{\mathrm{L} 0}$ the number of pulses within a resonator round-trip time $T=2 L / c$ ( $L$ resonator length) [16]. Curves 1 and 2 are calculated without etalon. The effect of two-photon absorption (curve 1) is slightly more pronounced than in case without background. The situation for the resonator with etalon is depicted by curves $1^{\prime}$ and $2^{\prime}$. The preferable amplification of 
background within the opening time of the absorber elongates the pulse duration as is clearly seen by the rise of pulse duration of curves $1^{\prime}$ and $2^{\prime}$ in the early part of the pulse train. It should be noticed that for pulses with background noise and short initial duration $\Delta t_{\mathrm{L} 0}<\tau_{\mathrm{R}}$, the transient bleaching of the dye elongates the temporal half-width $\left(\Delta t_{\mathrm{L}}>\Delta t_{\mathrm{L} 0}\right)$ and narrows the spectrum $\left(\Delta \nu_{\mathrm{L}}<\Delta \nu_{\mathrm{L} 0}\right)$ in the early part of the nonlinear region.

The experimental pulse durations are in reasonable agreement with the calculations. In case without etalon the curve 1 of fig. $4 \mathrm{c}$ applies to the experimental situation (circle). Secondary pulses need not be considered since they are outside the opening time of the dye. For the case of pulse generation with the etalon in the oscillator the curve $1^{\prime}$ of fig. $4 \mathrm{c}$ describe the experimental situation (triangle). When secondary pulses are resolved, the envelope of the pulse duration follows again curve $1^{\prime}$ of fig. $4 \mathrm{c}$ while the individual central pulse width approaches the background free curve $1^{\prime}$ of fig. $4 \mathrm{~b}$. The temporal pulse development in the trailing part of the pulse train is complicated by selfphase modulation [10] and spectral hole burning [11] which are not included in the theoretical analysis of fig. 4 .

\section{Conclusions}

The influence of partial suppression of natural mode selection on the duration of the generated pulses was discussed. The finite recovery time of the saturable absorber, the two-photon absorption of the active medium and the finite response of the active medium determine the observed pulse durations and hinder more pronounced pulse shortening along the pulse train by insertion of a properly adjusted etalon.

\section{Acknowledgement}

The authors are grateful to Th. Ascherl for technical assistance and to G. Pleininger for assistance during parts of the experiments. They thank the Rechenzentrum of the University for disposal of computer time.

\section{References}

[1] M.W. McGeoch, Optics Comm. 7 (1973) 116.

[2] S.V. Chekalin, P.G. Kryukov, Yu.A. Matveets and O.B. Shalberashvili, Opto-Electronics 6 (1974) 249.

[3] D. von der Linde and K.F. Rodgers, Optics Comm. 8 (1973) 91.

[4] E.M. Gordeev, P.G. Kryukov, Yu.A. Matveets, B.M. Stepanov, S.D. Fanchenko, S.V. Chekalin and A.V. Sharkov, Soviet J. Quantum Electron. 5 (1975) 129.

[5] H. Graener and A. Laubereau, Optics Comm. 37 (1981) 138.

[6] A. Penzkofer, to be published.

[7] P.C. Magnante, IEEE J. Quantum Electron. QE-8 (1972) 440.

[8] W. Demtröder, Laser spectroscopy. Basic concepts and instrumentation (Springer, Berlin, 1981) p. 152.

[9] E. Hecht and A. Zajac, Optics (Addison-Wesley, Reading, 1974) p. 74.

[10] R.C. Eckardt, C.H. Lee and J.N. Bradford, OptoElectronics 6 (1974) 67.

[11] A. Penzkofer and N. Weinhardt, IEEE J. Quantum Electron. QE-19 (1983) 567.

[12] A. Seilmeier, B. Kopainsky and W. Kaiser, Appl. Phys. $22(1980) 355$.

[13] A. Penzkofer and W. Kaiser, Appl. Phys. Letters 21 (1972) 427.

[14] A. Penzkofer and W. Falkenstein, Chem. Phys. Letters 44 (1976) 547.

[15] J. Herrmann, F. Weidner and B. Wilhelmi, Appl. Phys. B26 (1981) 197.

[16] W.H. Lowdermilk, in: Laser handbook, Vol. 3, ed. M.L. Stitch (North-Holland, Amsterdam, 1979) p. 361. 